\title{
Effect of Apple Quality, Yeast Strains and Use of Antimicrobial Additives on Cider Production with Therapeutic Potential
}

\section{Mujahid Hassan Khan ${ }^{1 *}$, Ayesha Kiran², Hina Saif ${ }^{3}$, Muhammad Saqib Nadeem $^{4}$ and Muniba Khan ${ }^{2}$}

${ }^{1}$ Food Tech Department, Chulalongkorn University, Bangkok, Thailand

${ }^{2}$ Food Science and Technology Department, Govt. College University, Faisalabad, Pakistan

${ }^{3}$ College of Food Science \& Technology, Nanjing Agricultural University, Nanjing, China

${ }^{4}$ NIFSAT, University of Agriculture, Faisalabad, Pakistan

*Corresponding Author: Mujahid Hassan Khan, Food Tech Department,

Chulalongkorn University, Bangkok, Thailand.
Received: August 05, 2021

Published: December 31, 2021

(C) All rights are reserved by Mujahid Hassan

Khan., et al.

\begin{abstract}
The aim of this review was to evaluate the effect of apple quality on cider production and to determine the therapeutic potentials of the apple cider. Apple Cider possesses antioxidant property, and it is highly beneficial in Type 2 Diabetes and environmentally induced malignancies. Modifiable risk factors and behavioral modifications, such as eating habits, are reported to prevent 30-40\% of cancer cases. Basically, the effect of yeast strains on cider, effects of cultivars on cider production and therapeutic potentials including anti-carcinogenic effect, antimicrobial activity, hypocholesterolemic effect, hypolipidemic effect and glycemic control, antioxidant activity are reviewed.
\end{abstract}

Keywords: Yeast; Diabetes; Apples

\section{Introduction}

Apples are well-known for their nutritional value. Consumers are familiar with the proverb "an apple a day keeps the doctor away," and scientific study backs it up by demonstrating the existence of several bioactive chemicals, such as phenolic chemicals, Cancer risk reduction, high antioxidative power, anti-inflammatory and anti-tumor capabilities, and suppression of carcinogenesis in skin, breast, and colon are all health benefits connected with polyphenols in apples [1,2]. Apple cider has recently been one of the most widely discussed topics among the researchers and home remedy seekers. The effects of apple cider have been researched for hundreds of years. Hippocrates, the father of modern medicine, is said to have created a mixture of honey and apple cider for the treatment of different ailments 5000 years ago, in 400 B.C. It was said to have been used for cleansing troops' wounds during the American Civil War [3]. Apple cider is prepared by crushing apples and then adding bacteria and yeast to begin the alcoholic fermentation process. The carbohydrates are turned to alcohol, which is then turned to vinegar by acetic acid-forming bacteria in the second fermentation phase (Acetobacter) [4].

Apples are particularly sensitive to fungal contamination, which can occur at many stages: it can happen before harvest, harming the tree in the orchard, while the most severe diseases that cause fruit spoiling happen after harvest. Fungal postharvest infections cause major economic losses in apple production, with losses ranging from 30-40 percent in impoverished nations to up to 60 percent in the most acute cases [5].

Cider is commonly thought of as an apple-based beverage. In North America, the phrase "cider" refers to unpasteurized, hazy apple juice, whereas "hard cider" refers to a fermented product. However, in Europe, the name "cider" refers to a fermented alcoholic beverage with an alcohol level of 1.2 to 8.5 percent $\mathrm{v} / \mathrm{v}$ [6]. Pressed apple juice is used to make apple cider. Only a small percentage of 
phenolic chemicals are extracted during fruit crushing, pressing, and juice extraction. This is primarily due to the removal of the peel and seeds, both of which are high in phenolic chemicals.

The quality of hazy apple juice, cider, and vinegar is influenced by a number of factors, including cultivar, ripeness, and the microorganisms used for fermenting. Sweeter goods were chosen to a great amount for juices and ciders, and the TSS/TA ratio appears to be a good predictor of customer acceptability. In this study, an apple cultivar that generated good juice also made good cider, but the cultivar had less of an impact on vinegar production [7].

EHEC is a kind of Shiga toxin-producing Escherichia coli that causes hemorrhagic colitis and hemolytic uremic syndrome (HUS) in humans. The capacity of EHEC to cause fatal food-borne diseases with a low infectious dosage makes it a significant public health problem. Foodborne infections caused by EHEC are projected to cost the US economy $\$ 200$ million each year [8]. Phytochemicals are a class of natural, plant-derived molecules that have been utilised as food preservatives and flavour enhancers for centuries. Phytochemicals have been found to have a wide range of therapeutic qualities, including antiseptic, anti-inflammatory, antioxidant, and antibacterial activities, according to several studies [9]. Because of their large margin of safety and growing concern about the safety of synthetic chemicals, the use of phytochemicals as food antimicrobials has gotten a lot of interest in the last decade [10]. The use of infected cattle manure in apple orchards is the most common source of EHEC contamination in apple cider. In the past, EHEC contamination in cider was caused by using "drop" or "windfall" apples infected with the disease via animal faeces in orchard soil.

\section{Factors contributing the quality}

There are many factors that contribute to the quality status in cider production that may include apple cultivar type used for the production of cider, post-harvest conditions provided to the apple and its effects on chemical composition and yeast strains effect on the chemical composition of the apple cider. However, the use of antimicrobial agents is an emerging technique to prevent many of these factors. In the pre-harvest, harvest, and postharvest periods, apples are very sensitive to fungal infestation. Postharvest fungal infections cause significant economic losses in apple production. When mouldy apples are ready for processing, mycotoxins accumulate in the fruit, posing a significant risk.

\section{Fermented foods' probiotic properties}

Many species present in the fermented foods are similar or they share physiological characteristics with the species that are beneficial to gastrointestinal health. The idea that living microorganisms associated with food fermentations might perform positive activities in the GI tract is in line with the growing consensus that probiotic cultures' primary health benefits can be attributed to a species rather than individual strains of a species [11].

Ingestion of live fermentation-associated microorganisms might thus have a comparable effect on intestinal epithelial, immunological, and enteroendocrine cells as probiotic strains. Until previously, these effects were mostly ascribed to specific strains [12].

Because of their high nutritional value in terms of vitamins, minerals, dietary fibre, and antioxidant chemicals, fruit juices have been recommended as an alternate carrier for LAB fermentation. Fermented fruits with LAB, thus, can be a good and nutritious alternative functional food, including probiotics and providing improved nutritional, physical-chemical, and sensory qualities [13].

\begin{tabular}{|c|c|}
\hline \multicolumn{2}{|c|}{$\begin{array}{r}\text { Food fermentations using probiotic microbes, whether } \\
\text { traditional or novel }\end{array}$} \\
\hline $\begin{array}{c}\text { Species that have been } \\
\text { shown to have probiotic } \\
\text { function }{ }^{1}\end{array}$ & $\begin{array}{c}\text { Fermented foods with high cell } \\
\text { counts of species strains that can be } \\
\text { counted on a regular basis }\end{array}$ \\
\hline Lactobacillus acidophilus & NSLAB long ripened cheese \\
\hline Lactobacillus fermentum & $\begin{array}{c}\text { Bushera, ting and other African } \\
\text { cereal porridges and beverages }\end{array}$ \\
\hline Lactobacillus paracasei & $\begin{array}{c}\text { Kvass, Salami' } \text {, NSLAB and in long- } \\
\text { ripened cheese }\end{array}$ \\
\hline Lactobacillus casei & NSLAB long-ripened cheese \\
\hline Lactobacillus rhamnosus & 'Villi', fermented oatmeal \\
\hline
\end{tabular}

Table 1: NSLAB, non-starter lactic acid bacteria.

${ }^{1}$ According to Health Canada.

${ }^{2}$ If the existence of a probiotic organism is contingent on its inclusion as a competitive starting culture, the fermented food is printed in bold.

Source: [14]. 
Effect of apple cultivars on cider quality

Each apple cultivar has a unique polyphenolic profile that is influenced by harvest year, environmental factors, cultivation, and storage conditions [15]. The phenolic content and profile of apple ciders have a significant impact on the sensory qualities of the beverage, particularly colour, bitterness, and astringency [16]. The yeast and fermentation conditions used have a significant impact on the development of sensory qualities in ciders. Because diverse yeast strains are widely available, both yeast manufacturers and apple cider producers are interested in monitoring their impact on apple cider content and phenolic profile. Malolactic fermentation (the conversion of L-malic acid to L-lactic acid and carbon dioxide) is a technique used in the cider making process to minimise sourness.

Basically the apple cultivars impacts on the phenolic composition of the cider. The apple cultivars plays a major effect on phenolic composition, underlining and confirming the previously recognised importance of cultivar selection in cider production. Estonian apple cultivars having the potential to be utilised in ciders were considered in a study [2]. The phenolic composition of the apple was influenced by its ripening stage, which had a minor but significant impact [7]. Unripe apples have higher levels of phenolic chemicals than ripe apples, though the effect varies by cultivar. Typically, ripe or overripe apples are utilised in cider production because of their softened structures, which result in increased juice yields and a rise in sugar content as the apples ripen [17]. According to a study, less ripe apples can be used to make ciders with much higher phenolic content. The less ripe apples can also be blended with other cultivars to boost the amount of phenolic chemicals in the finished cider. By differing in the release of free Hydroxycinnamic acids from the matching quinic acid esters, the commercial yeast products employed in this investigation had only a little impact on the phenolic profiles. However, due to the probable release of volatiles bound by ester bonds, this impact may result in a change in flavour character [18].

\section{Effect of yeast strain on chemical composition}

A number of fungi have been identified as the cause of apple illnesses. Despite the fact that geographical variances have been found and that a variety of factors, such as meteorological conditions, agricultural practises, cultivars, and the postharvest environment, may influence the apple mycota composition, the

\begin{tabular}{|c|c|}
\hline \multicolumn{2}{|c|}{ Organic Acids in Apple Cider } \\
\hline Compound & Concentration (g/L) \\
\hline Acetic Acid & 50.9 \\
\hline Citric Acid & 0.02 \\
\hline Formic Acid & 0.28 \\
\hline Lactic Acid & 0.38 \\
\hline Malic Acid & 3.56 \\
\hline Succinic Acid & 0.27 \\
\hline Fructose & 6.83 \\
\hline Acetoin & 0.21 \\
\hline 2,3-Butanediol & 0.37 \\
\hline Ethanol & 1.03 \\
\hline Ethyl acetate & 0.14 \\
\hline
\end{tabular}

Table 2

Source: [19].

most important diseases have been recorded worldwide [20]. In the field and during storage, fungi are the principal disease agents of apples. The severity of post-harvest infections during storage can result in significant yield and fruit quality losses, and several measures for controlling them have been examined [21]. Because fruit intended for processing is generally of lower quality than fruit intended for direct consumption as fresh fruits, the potential impacts of fungal infections on juice processing products has received far too little consideration. With the exception of the presence of mycotoxins in cider, this is true [22]. The infection has the potential to drastically alter the qualities of the fruit and its juice [23]. Most of them start colonization when there are sores or physical damage in the skin, which can be caused by insects or birds in the field, or by poor postharvest handling. One of the most commonly reported pathogens is Penicillium expansum, which causes blue rot o blue mould. Botrytis cinerea (grey rot), Monilinia fructigena and other Monilinia spp. (brown rot), Colletotrichum spp. (bitter rot), Mucor spp., and Rhizopus spp. are some of the more frequent ones. These aggressive pathogens can impact negatively on fruit both before and after harvest [5,24]. Although some of these fungus can cause damage to the fruit's interior, they're more commonly connected with surface lesions. They are normally controlled by applying fungicides prior to harvest and preventing further physical damage to the fruits during the postharvest period [25]. It was previously shown that different fungal pathogens, such 
as Botrytis cinerea, Penicillium expansum, Mucor piriformis, and Monilinia sp., produce different volatile compounds in infected apples [26]. During storage in refrigerated chambers, the incidence and severity of some fungal diseases, as well as the accumulation of mycotoxin, increases.

Penicillium and Alternaria are two of the most common apple diseases that can produce mycotoxins. Apple-based food has been found to have both penicillium expansum patulin and Alternaria toxins. Mycotoxin contamination poses a significant risk, particularly in items designed for children's ingestion, such as apple-based baby food [25].

Several investigations have found significant changes in the chemical composition of ciders made from sound apples vs ciders made from infected apples. Furthermore, the scent composition of ciders made from infected apples varied due to differences in the concentration of numerous molecules belonging to each chemical group discovered. Because alcohols, esters, and acids are essential components of cider aroma [21,27].

Basically the yeast affects the volatile composition of the apple cider. The yeast used in fermented beverage production contributes to the final fragrance profile by increasing the levels of higher alcohols and esters [28]. The potential of mycotoxin buildup in the fruit is another significant drawback of fungal contamination. When mycotoxigenic species from genera like Penicilium, Alternaria, or Fusarium cause apple rotting, the danger of mycotoxin contamination is substantial, and this danger is amplified if these toxigenic species may develop during storage at refrigerated temperatures [29]. Apple concentrates are often processed throughout the year, which necessitates keeping the fruit refrigerated for several months. In the case of apples, cold storage in a controlled environment can last anywhere from 9 to 12 months, depending on the apple variety and destination. Several infections that have invaded the apple's inside are capable of developing symptoms during storage, and in certain cases, even synthesising mycotoxins during this time. Despite the fact that certain process stages have been shown to minimise toxin concentrations [30].

The yeast and fermentation environment used have a significant impact on the development of sensory qualities in ciders. Because diverse yeast strains are widely available, both yeast manufacturers and apple cider producers are interested in monitoring their impact on apple cider contents and phenolic profile. Malolactic fermentation (the conversion of L-malic acid to L-lactic acid and carbon dioxide) is a technique used in the cider-making process to minimise sourness. Using multivariate statistical models, the researchers compared the phenolic profiles of apple juices to those of ciders after yeast fermentation and malolactic fermentation.

\section{Effect of antimicrobial additives}

Freshly pressed apple cider is a ready-to-drink beverage that is frequently processed without microbial inactivation procedures. Despite the fact that pasteurisation is an FDA-approved method for reducing pathogens in apple cider, concerns about its negative effects on cider taste and texture, as well as its affordability for small cider producers, have sparked interest in looking into alternative methods for improving its microbiological safety. Furthermore, typical preservatives like sorbates and benzoates have been proven to have just a minor effect on EHEC in apple cider. As a result, research into the efficiency of various naturally occurring antimicrobial compounds for suppressing infections in foods has sparked. As a result, many phytochemicals, such as carvacrol, cinnamaldehyde, and p-cymene, as well as caffeic acid, have been investigated for their effect on EHEC survival in apple juice or cider [31]. A study suggested that mega-resveratrol (RV) and naringenin (NG) could be used as efficient antibacterial ingredients in apple cider to inactivate EHEC. However, organoleptic investigations are needed to determine whether cider enhanced with these natural molecules is acceptable to consumers [32]. It could be a high blood glucose level while you're hungry and after you eat a meal. In type 1 diabetes, hyperglycemia can result from the death of the beta cell, which leads in a lack of insulin. Insulin is present in type 2 diabetes, but tissues are resistant to it, resulting in elevated blood glucose levels. Vinegar enhanced insulin sensitivity by 19 percent in type 2 diabetes and 34 percent in a person with pre-diabetes [33]. The dose and type of vinegar can affect postprandial blood glucose and insulin response. In healthy people, using vinegar with carbohydrate-rich foods is more beneficial than using vinegar with low-glycaemic foods. Meal oxidative stress is not only improved by adding vinegar to a standard meal, but it also lowers postprandial glucose levels and lengthens the satiety period, which should help to reduce food cravings and lower calorie intake by consuming 1 to 2 spoons of vinegar with high glycemic index foods like white

Citation: Mujahid Hassan Khan., et al. "Effect of Apple Quality, Yeast Strains and Use of Antimicrobial Additives on Cider Production with Therapeutic Potential". Acta Scientific Microbiology 5.1 (2022): 94-103. 
bread, white rice, and other high glycemic index foods, which lowers random blood glucose by $25 \%$ [34]. Whole apple cider vinegar is more effective than acetic acid alone in the control of diabetes. Consumption of "mother of vinegar" may be more beneficial than consumption without it. Acetic acid and chlorogenic acid have more promise for diabetes, lipid disorders, hypertension, and weight loss than the other components of ACV [35]. When random blood sugar and insulin values were measured after 2 hours of meal intake, vinegar was more effective in high glycaemic index meal (low-fat milk and mashed potato) than in low glycemic index meal (lowfat cheese, whole grain, lettuce, etc.) with the same composition of nutrients in iso-caloric meal [35].

\section{Therapeutic potential}

\section{Anti-carcinogenic effect}

Apple polyphenols comprise catechin, epicatechin, flavonoids, epicatechin, and procyanidins which inhibit colon cancer cells considerably [36]. 8 Apple cider vinegar polyphenols cause cytotoxicity in human urinary bladder cancer cells (TSGH-8301), which is linked to oxidative stress and apoptosis [37]. Clarissa Gerhauser [38] published a review on the cancer chemoprotective potential of apple components and apple juice in 2008. In vivo animal studies as well as clinical data were included in this review, which revealed that apple products had a cancer-targeted mechanism. In 2005, Barth., et al. [39] used a well-established rodent model to persuade colon damage associated with colon cancer to be caused by 1,2-dimethylhydrazine, and discovered that cloudy apple juice reduced DNA damage, hyperproliferation, and acted as a cancerpreventative agent. Pelingo apple juice inhibited breast cancer cell proliferation and caused cell accumulation in the G2/M phase of the cell cycle. In addition, apple juice reduced tumour genesis in distinct cell lines generated by 12-o-tetra-decanoylphorbol-13acetate (TPA).

\section{Antimicrobial activity}

In a study the antimicrobial activity of apple cider was studies against the some strains of bacteria and yeast including Escherichia coli, Staphylococcus aureus, Salmonella paratyphi A, Salmonella paratyphi $B$ and two fungi Aspergillus niger and Candida albicans. The results revealed that apple cider is potent antimicrobial agent against gram positive and gram negative as well and also it was found that it worked very well against the both fugal strains [40]. Because apple cider is high in natural enzymes, it can aid in the elimination of candida in the body. Candida has been linked to a variety of health problems, including memory loss, fatigue, migraines, depression, sugar cravings, and yeast infections. Although it is a naturally occurring yeast, it can quickly develop out of control if the body becomes excessively acidic as a result of consuming too much processed foods or sugar, or if healthy bacteria is driven off as a result of antibiotic use. Apple cider is fermented with a beneficial yeast that acts as a prebiotic for good bacteria in the gut, essentially assisting in the growth of good bacteria as you eliminate the items that feed dangerous yeasts like candida [41].

\section{Hypocholesterolemic effect}

Cardiovascular disease (CVD) is the world's leading cause of death. Cardiovascular disease can be caused by atherosclerosis, high blood cholesterol levels, a poor diet, high blood pressure, smoking, obesity, and diabetes.

\section{Hypolipidemic effect and glycemic control}

Dyslipidemia is usually connected with type 2 diabetes. Attempting to reach normal blood levels of total cholesterol, low density lipoprotein (LDL), high density lipoprotein (HDL), and triglycerides is a part of managing the condition. Atherosclerosis is strongly linked to dyslipidemia [35]. Impairment of insulin secretion, hepatic glucose overproduction and insulin resistance are the main causes of diabetes. Insulin resistance is a crucial factor in the development of diabetes, but it also plays a role in hypertension, atherosclerosis, and dyslipidemia [42]. Polyphenols have distinct nutraceutical and supplemental therapy qualities for diabetes mellitus type 2 in a variety of ways. Polyphenols, which include lingams stilbenes, phenolic acids, and flavonoids, have been shown to have potential for reducing diabetes-related diseases and complications. Polyphenols found in plants and polyphenol-rich foods lower blood sugar, insulin resistance, and increase adipose tissue metabolism, dyslipidemia and carbohydrate modulation, and reduce oxidative stress. Polyphenol compounds can help to avoid long-term diabetic problems such as cardiovascular disease, retinopathy, neuropathy, and nephropathy [43]. In several clinical trials, apple cider vinegar has been shown to lower obesity, hyperglycemia, hyperlipidemia, and hyperinsulinemia, according to the complete review. Several mechanisms for apple cider vinegar's therapeutic effects have been proposed, including delayed gastric emptying, increased glucose utilisation and enteral absorption, up-regulation of flow-mediated

Citation: Mujahid Hassan Khan., et al. "Effect of Apple Quality, Yeast Strains and Use of Antimicrobial Additives on Cider Production with Therapeutic Potential". Acta Scientific Microbiology 5.1 (2022): 94-103. 
vasodilation, suppression of hepatic glucose production, increase in lipases, decrease in lipogenesis, insulin secretion facilitation, faecal bile acid execration stimulation, and increased energy expenditure, to name a few.

Despite these findings, more clinical trials are required [44].

Another route of vinegar's antiglycemic impact is enteral carbohydrate absorption; vinegar reduces disaccharide activity rather than glucose transport inactivation in intestinal cells. A 15-day treatment with acetic acid suppressed sucrose, maltose, lactose, and trehalase in vitro, but acetic acid had no effect on sucrose or maltase de novo synthesis either at the translational or transcriptional level, implying that suppression occurs during post-translational processing, with acetic acid invading posttranslational like intracellular trafficking [45]. Acetic acid interferes with the digestion of starch molecules, reducing the quantity of glucose absorbed into the bloodstream after a meal.

Many other research have found that drinking apple cider vinegar helps to lower blood sugar levels after a high-carbohydrate breakfast [46].

\section{Anti-diabetic effect}

Diabetes mellitus is a metabolic condition marked by elevated blood sugar levels caused by decreased insulin resistance, insulin secretion, and hepatic glucose overproduction [47]. [48] reported in 2008 that ACV has a protective impact on lipid profile in both diabetic and normal rats. In rat models, apple-derived products improved hyperglycemia, hyperinsulinemia, body weight gain, and other metabolic diseases via modulating the gut flora [49]. According to the Meta analysis, the consumption of apple products (apple juice, sauce) was linked to a lower prevalence of obesity [50].

Fathy and Drees [49] investigated the effects of hazy apple juice and apple peel extract on the rat pancreas in 2016. Apple juice induced antihyperglycemic benefits by reducing inflammation, modifying oxidative stress, and lowering lipid profile, suggesting that it could be a suitable target for diabetes mellitus.

\section{Antioxidative properties}

Compositional analysis of apple cider shows that apple cider contains considerable amount of total phenolic compounds. A study was conducted in 2015 and the results of the analysis TEAC and ORAC reveals that the chemically apple cider is potent against oxidizing agents [51]. So, that's the reason it have also a positive effect on the blood anti-oxidative actions and helps detoxification of the body by neutralizing the oxidants.

\section{Anti-cardiotoxicity properties}

Atherosclerosis is a chronic disease caused by inflammatory activation and oxidative stress, and it leads to heart and circulatory problems. In 2018, a study was proposed, and the results showed that cardiovascular health products had potential impacts with apple cider vinegar in improving atherogenesis, reducing triacylglycerol in mice blood, and ameliorating inflammation [52]. In $\mathrm{H} 9 \mathrm{c} 2$ cells, polyphenolic substances found in apple peel extract decreased arsenic trioxide-induced cardiotoxicity. SOD, catalase, glutathione, GSH (Glutathione reductase), and caspase 3 activity were all affected by the extract [53].

\section{Management of hypertension}

Diabetes can damage the autonomic nervous system and endothelium, causing microvascular complications and impairing blood flow autoregulation. Diabetics have been found to have lower amounts of the vasodilator nitric oxide and higher levels of the vasoconstrictor endothelin-1, resulting in vasoconstriction [54]. Vascular damage, which leads to cardiovascular disease, is a result of high blood pressure. When compared to controls and participants who simply consumed vinegar, acetic acid coupled with vinegar was found to dramatically drop blood pressure $21-30 \mathrm{mmHg}$ lower than the control) and renin activity. Lower blood pressure could be due to a decrease in renin and the consequent release of angiotension II. There was also a drop in aldosterone. At a concentration of $46.2 \mathrm{~g} / \mathrm{L}$, rice vinegar and acetic acid were administered [55]. Rice vinegar contains about 4\% acetic acid [35]. As a result, acetic acid administered alone would be more effective than vinegar solution. It has been proposed that acetic acid increases calcium absorption, causing calcium influx into renin secretory cells and limiting renin secretion [55]. Angiotensin-converting enzyme activity was also found to be reduced by a mixture of red wine vinegar and grape juice (ACE).

\section{Management of obesity}

Obesity is the world's most serious health problem, and it has reached epidemic proportions. New targets have been established 
to recognise the molecules that govern the distribution, organisation, and breakdown of adipose tissue, which will aid in the prevention and treatment of obesity. Advances in food science and nutrition research have revealed the possibility of balancing certain physiological processes and molecular signalling in humans using food-derived components, with the goal of controlling and slowing obesity growth at the molecular level.

Obesity is usually related with type 2 diabetes, and weight loss is considered an important component in diabetes management [56]. It's been suggested that acetic acid can help you eat less. This could be due to the acetic acid in vinegar's taste and the nausea it can cause when consumed [57]. A study found that adding white vinegar to a meal boosted the subjective rating of satiety when compared to a control group. Satiety improvements may lead to reduced food consumption and, as a result, weight loss [58]. In a 12 -week trial, it was discovered that consuming $75 \mathrm{~g}$ and $150 \mathrm{~g}$ of acetic acid significantly lowered bodyweight, BMI, visceral fat, and waist circumference when compared to a control group [59]. Chlorogenic acid has been shown to block the G1 phase of the cell cycle in mouse embryo 3T3-L1 preadipocytes, limiting proliferation. At a concentration of $100 \mathrm{M}$, preadipocytes were suppressed in this investigation in a time and dose-dependent manner. One strategy for reversing obesity is to reduce preadipocyte development [60]. Another recent study found that adding 0.02 percent $(\mathrm{w} / \mathrm{w})$ chlorogenic acid to the diet of mice on a high fat diet resulted in a substantial 16 percent weight loss compared to the control group and elevated adiponectin levels [61].

\section{Future prospective}

There is an opportunity to properly analyze the properties of the apple cider and to interpret the health benefits and chemical composition. A considerable work is already done worldwide on it but still there is a room to have a look over it deeply.

\section{Conclusion}

Applecideris anatural healthfood thathasa number ofalternative health advantages. It has a significant anti-cancer, antioxidant, antiinflammatory, and hepatoprotective effect due to the presence of different polyphenolic chemicals. The antioxidant mechanism identified crucial indications for apple cider potential to protect against cardiovascular disease, cancer, diabetes, and asthma. Apple cider is an easily accessible commodity that may be simply included into meals. Its beneficial characteristics as a whole product, as well as the capacities of its component constituents, have been established by a huge number of studies.

\section{Bibliography}

1. Francini A and LJA Sebastiani. "Phenolic compounds in apple (Malus x domestica Borkh): compounds characterization and stability during postharvest and after processing". Antioxidants 2.3 (2013): 181-193.

2. Laaksonen 0., et al. "Impact of apple cultivar, ripening stage, fermentation type and yeast strain on phenolic composition of apple ciders". Food Chemistry 233 (2017): 29-37.

3. Gopal J., et al. "Authenticating apple cider vinegar's home remedy claims: antibacterial, antifungal, antiviral properties and cytotoxicity aspect". Natural Product Research 33.6 (2019): 906-910.

4. Sheng TJJAYSJ. "Viability of heart vein opening drink". APEC Youth Scientist Journal 6.2 (2014): 231-247.

5. Köhl J., et al. "Dynamics of post-harvest pathogens Neofabraea spp. and Cadophora spp. in plant residues in Dutch apple and pear orchards". Plant Pathology 67.6 (2018): 1264-1277.

6. Lea AG and JF Drilleau. "Cidermaking, in Fermented beverage production". Springer (2003): 59-87.

7. Heikefelt C. "Chemical and sensory analyses of juice, cider and vinegar produced from different apple cultivars". Plant Breeding and Biotechnology (2011).

8. Hoffmann S., et al. "Economic burden of major foodborne illnesses acquired in the United States". Economic Research Service EIB-140. (2015).

9. Nair DV., et al. "Food grade Pimenta leaf essential oil reduces the attachment of Salmonella enterica Heidelberg (2011 ground turkey outbreak isolate) on to turkey skin". Frontiers in Microbiology 8 (2017): 2328.

10. Salamci E., et al. "Chemical compositions, antimicrobial and herbicidal effects of essential oils isolated from Turkish Tanacetum aucheranum and Tanacetum chiliophyllum var. chiliophyllum". Biochemical Systematics and Ecology 35.9 (2007): 569581. 
11. Hill C., et al. "Expert consensus document: The International Scientific Association for Probiotics and Prebiotics consensus statement on the scope and appropriate use of the term probiotic". Nature Reviews Gastroenterology and Hepatology (2014).

12. Gao C., et al. "Histamine $\mathrm{H} 2$ receptor-mediated suppression of intestinal inflammation by probiotic Lactobacillus reuteri". mBio 6.6 (2015): e01358-15.

13. Guiné RP., et al. "Apple fermented products: an overview of technology, properties and health effects". Processes 9.2 (2021): 223.

14. Marco ML., et al. "Health benefits of fermented foods: microbiota and beyond". Current Opinion in Biotechnology 44 (2017): 94-102.

15. Thompson-Witrick, K.A., et al. "Characterization of the polyphenol composition of 20 cultivars of cider, processing, and dessert apples (Malus× domestica Borkh) grown in Virginia". Journal of Agricultural and Food Chemistry 62. 41 (2014): 10181-10191.

16. Ye M., et al. "Evolution of polyphenols and organic acids during the fermentation of apple cider". Journal of the Science of Food and Agriculture 94.14 (2014): 2951-2957.

17. Malec M., et al. "Polyphenol profiling of a red-fleshed apple cultivar and evaluation of the color extractability and stability in the juice". Journal of Agricultural and Food Chemistry 62. 29 (2014): 6944-6954.

18. Makila L., et al. "Stability of hydroxycinnamic acid derivatives, flavonol glycosides, and anthocyanins in black currant juice". Journal of Agricultural and Food Chemistry 64.22 (2016): 4584-4598.

19. Caligiani A., et al. "Identification and quantification of the main organic components of vinegars by high resolution $1 \mathrm{H}$ NMR spectroscopy". Analytica Chimica Acta 585.1 (2007): 110-119.

20. Sun H., et al. "Antioxidant and prebiotic activity of five peonidin-based anthocyanins extracted from purple sweet potato (Ipomoea batatas (L) Lam)". Scientific Reports 8.1 (2018): 1-12.

21. Valiuškaitė A., et al. "Disease incidence on different cultivars of apple tree for organic growing". Agronomy Research 7.1 (2009): 536-541.
22. Zhong L., et al. "Patulin in apples and apple-based food products: The burdens and the mitigation strategies". Toxins 10.11 (2018): 475.

23. Gao L., et al. "Etiology of moldy core, core browning, and core rot of Fuji apple in China". Plant Diseases 97.4 (2013): 510516.

24. Wenneker M., et al. "Control of postharvest storage rots of apples and pears in The Netherlands". in III International Symposium on Postharvest Pathology: Using Science to Increase Food Availability". Acta Horticulturae 1144. (2015).

25. Patriarca AJCOiFS. "Fungi and mycotoxin problems in the apple industry". Current Opinion in Food Science 29 (2019): 4247.

26. Gong D., et al. "A comparison of postharvest physiology, quality and volatile compounds of 'Fuji'and 'Delicious' apples inoculated with Penicillium expansum". Postharvest Biology and Technology 150 (2019): 95-104.

27. Simonato B., et al. "Effects of post-harvest fungal infection of apples on chemical characteristics of cider". Lebensmittel-Wissenschaft und-Technologie 138 (2021): 110620.

28. McKay M., et al. "Fermented beverages: Beers, cidars, wines and related drinks". (2011).

29. Welke JE., et al. "Effect of processing stages of apple juice concentrate on patulin levels". Food Control 20.1 (2009): 48-52.

30. Oteiza JM., et al. "Influence of production on the presence of patulin and ochratoxin $\mathrm{A}$ in fruit juices and wines of Argentina". LWT 80 (2017): 200-207.

31. Baskaran SA., et al. "Inactivation of Escherichia coli 0157: H7 in apple juice and apple cider by trans-cinnamaldehyde". International Journal of Food Microbiology 141.1-2 (2010): 126129.

32. Nair MS., et al. "Inactivation of Escherichia coli 0157: H7 in apple cider by resveratrol and naringenin". Journal of the American College of Cardiology 86 (2020): 103327.

33. Budak NH., et al. "Functional properties of vinegar". Concise Reviews in Food Science 79.5 (2014): R757-R764. 
34. O’Keefe JH., et al. "Dietary strategies for improving post-prandial glucose, lipids, inflammation, and cardiovascular health". Journal of the American College of Cardiology 51.3 (2008): 249255.

35. Morgan J., et al. "The potential of apple cider vinegar in the management of type 2 diabetes". International Journal of Diabetes Research 5.6 (2016): 129-134.

36. Hung C.-H., et al. "Apple polyphenol inhibits colon carcinoma metastasis via disrupting Snail binding to focal adhesion kinase". 12 (2015): 80-91.

37. Kao, Y.-L., et al. "Apple polyphenol induces cell apoptosis, cell cycle arrest at G2/M phase, and mitotic catastrophe in human bladder transitional carcinoma cells". Journal of Functional Foods 14 (2015): 384-394.

38. Gerhauser CJPm. "Cancer chemopreventive potential of apples, apple juice, and apple components". Planta Medica 74.13 (2008): 1608-1624.

39. Barth S., et al. "Cloudy apple juice decreases DNA damage, hyperproliferation and aberrant crypt foci development in the distal colon of DMH-initiated rats". Carcinogenesis 26.8 (2005): 1414-1421.

40. Saqib AJMJos. "Antimicrobial activity of apple cider vinegar". Mapana Journal of Sciences 16.2 (2017): 11-15.

41. Shankar V., et al. "A review on microbial degradation of drinks and infectious diseases: A perspective of human well-being and capabilities". Journal of King Saud University-Science. 33.2 (2021): 101293.

42. Ali Z., et al. "A double-blinded, randomized, placebo-controlled study evaluating the impact of dates vinegar consumption on blood biochemical and hematological parameters in patients with type 2 diabetes". Tropical Journal of Pharmaceutical Research 17.12 (2018): 2463-2469.

43. Bahadoran Z., et al. "Dietary polyphenols as potential nutraceuticals in management of diabetes: a review". Journal of Diabetes and Metabolic Disorders 12.1 (2013): 1-9.

44. Petsiou EI., et al. "Effect and mechanisms of action of vinegar on glucose metabolism, lipid profile, and body weight". Nutrition Reviews 72.10 (2014): 651-661.
45. Johnston CS and CAJMGM Gaas. "Vinegar: medicinal uses and antiglycemic effect". MedGenMed 8.2 (2006): 61.

46. Omar NAA., et al. "Hepatoprotective and antidiabetic effects of apple cider vinegar (a prophetic medicine remedy) on the liver of male rats". The Egyptian Journal of Hospital Medicine 62.1 (2016): 95-104.

47. Mahmoodi M., et al. "The effect of white vinegar on some blood biochemical factors in type 2 diabetic patients". Academic Journals 4.1 (2013): 1-5.

48. Shishehbor F., et al. "Apple cider vinegar attenuates lipid profile in normal and diabetic rats". Journal of Biological Sciences 11.23 (2008): 2634-2638.

49. Fathy SM and EAJBc. "Protective effects of Egyptian cloudy apple juice and apple peel extract on lipid peroxidation, antioxidant enzymes and inflammatory status in diabetic rat pancreas". BMC Complementary Altern Medicine 16.1 (2015): $1-14$.

50. Nicklas TA., et al. "Consumption of various forms of apples is associated with a better nutrient intake and improved nutrient adequacy in diets of children: National Health and Nutrition Examination Survey 2003-2010". Food and Nutrition Research 59.1 (2015): 25948.

51. Budak NH., et al. "Antioxidant activity and phenolic content of apple cider". Turkish Journal of Agriculture - Food Science and Technology 3.6 (2015): 356-360.

52. Wu Z., et al. "Lipid profiling in serum from apolipoprotein Eknock out mice fed with different diets and its application to the study of the regulatory effect on lipid metabolism". Food and Function Journal 9.10 (2018): 5103-5114.

53. Tripathi S., et al. "Apple cider vinegar (ACV) and their pharmacological approach towards Alzheimer's disease (AD): A review". Indian Journal of Pharmaceutical Education and Research 54.2s (2020): s67-s74.

54. Dokken BBJDS. "The pathophysiology of cardiovascular disease and diabetes: beyond blood pressure and lipids". Diabetes Spectrum 21.3 (2008): 160-165.

55. Kondo S., et al. "Antihypertensive effects of acetic acid and vinegar on spontaneously hypertensive rats". Bioscience, Biotechnology, and Biochemistry 65.12 (2001): 2690-2694. 
56. Forslund, K., et al. "Disentangling type 2 diabetes and metformin treatment signatures in the human gut microbiota". Nature 528. 7581 (2015): 262-266.

57. Darzi J., et al. "Influence of the tolerability of vinegar as an oral source of short-chain fatty acids on appetite control and food intake". Randomized Controlled Trial 38.5 (2014): 675-681.

58. Östman E., et al. "Vinegar supplementation lowers glucose and insulin responses and increases satiety after a bread meal in healthy subjects". Randomized Controlled Trial 59.9 (2005): 983-988.

59. Kondo T., et al. "Vinegar intake reduces body weight, body fat mass, and serum triglyceride levels in obese Japanese subjects". Randomized Controlled Trial 73.8 (2009): 1837-1843.

60. Hsu CL., et al. "Inhibitory effect of phenolic acids on the proliferation of 3T3-L1 preadipocytes in relation to their antioxidant activity". Comparative Study 54.12 (2006): 4191-4197.

61. Cho AS., et al. "Chlorogenic acid exhibits anti-obesity property and improves lipid metabolism in high-fat diet-induced-obese mice". Food and Chemical Toxicology 48.3 (2010): 937-943.

\section{Assets from publication with us}

- Prompt Acknowledgement after receiving the article

- Thorough Double blinded peer review

- Rapid Publication

- Issue of Publication Certificate

- High visibility of your Published work

Website: www.actascientific.com/

Submit Article: www.actascientific.com/submission.php

Email us: editor@actascientific.com

Contact us: +919182824667

Citation: Mujahid Hassan Khan., et al. "Effect of Apple Quality, Yeast Strains and Use of Antimicrobial Additives on Cider Production with Therapeutic Potential". Acta Scientific Microbiology 5.1 (2022): 94-103. 